\title{
Sensor Selection via Convex Optimization
}

\author{
Siddharth Joshi* $\quad$ Stephen Boyd ${ }^{\dagger}$ \\ To appear, IEEE Transactions on Signal Processing, 2008. \\ Originally submitted November 2007.
}

\begin{abstract}
We consider the problem of choosing a set of $k$ sensor measurements, from a set of $m$ possible or potential sensor measurements, that minimizes the error in estimating some parameters. Solving this problem by evaluating the performance for each of the $\left(\begin{array}{c}m \\ k\end{array}\right)$ possible choices of sensor measurements is not practical unless $m$ and $k$ are small. In this paper we describe a heuristic, based on convex optimization, for approximately solving this problem. Our heuristic gives a subset selection as well as a bound on the best performance that can be achieved by any selection of $k$ sensor measurements. There is no guarantee that the gap between the performance of the chosen subset and the performance bound is always small; but numerical experiments suggest that the gap is small in many cases. Our heuristic method requires on the order of $\mathrm{m}^{3}$ operations; for $m=1000$ possible sensors, we can carry out sensor selection in a few seconds on a $2 \mathrm{GHz}$ personal computer.
\end{abstract}

\section{Introduction}

We study the problem of selecting $k$ sensors, from among $m$ potential sensors. Each sensor gives a linear function of a parameter vector $x$, plus an additive noise; we assume these measurement noises are independent identically distributed zero mean Gaussian random variables. The sensor selection, i.e., the choice of the subset of $k$ sensors to use, affects the estimation error covariance matrix. Our goal is to choose the sensor selection to minimize the determinant of the estimation error covariance matrix, which is equivalent to minimizing the volume of the associated confidence ellipsoid. One simple method for solving the sensor selection problem is to evaluate the performance for all $\left(\begin{array}{c}m \\ k\end{array}\right)$ choices for the sensor selection, but evidently this is not practical unless $m$ or $k$ is very small. For example, with $m=100$

${ }^{*}$ Contact information for S. Joshi $\left({ }^{*}\right.$ corresponding author): Address: 350 Serra Mall, Packard 243, Stanford CA 94305, USA; Tel: (650) 723-9833; Fax: (650) 723-8473.

${ }^{\dagger}$ The authors are with the Department of Electrical Engineering, Stanford University (e-mail: \{sidj, boyd\}@stanford.edu). 
potential sensors, from which we are to choose $k=25$, there are on the order of $10^{23}$ possible choices, so direct enumeration is clearly not possible.

In this paper we describe a new method for approximately solving the sensor selection problem. Our method is based on convex optimization, and is therefore tractable, with computational complexity growing as $m^{3}$. For $m=1000$, the method can be carried out in a few seconds, on a $2 \mathrm{GHz}$ personal computer; for $m=100$, the method can be carried out in milliseconds. The method provides both a suboptimal choice of sensors, and a bound on the performance that can be achieved over all possible choices. Thus, we get a suboptimal design, and a bound on how suboptimal it is. Numerous numerical experiments suggest that the gap between these two is often small. Our basic method can be followed by any local optimization method. We have found, for example, that a greedy algorithm that considers all possible swaps between the set of selected and unselected sensors, accepting any swaps that improve the objective, can give a modest improvement in the quality of the sensor selection. When this local search terminates it gives a 2-opt sensor selection, i.e., one for which no swap of a selected and an unselected sensor has better objective value.

Prior and related work. The sensor selection problem arises in various applications, including robotics [HM97, sensor placement for structures Kam91, KP02, target tracking WYPE04, IB05, chemical plant control [KP99], and wireless networks [ZG04]. Sensor selection in the context of dynamical systems is studied in, e.g., GCHM06, KP98, Osh94. Sensor selection, with a rather different setup from ours, has been studied in sensor network management $\mathrm{REJ}^{+}$07, hypothesis testing in a sensor network [DLT02, and discrete-event systems JKG03. The sensor selection problem formulation we use in this paper can be found in, e.g., YSK93]. The sensor selection problem (and various extensions described in \$5) can be formulated in an information theoretic framework [CHZ02, MDW95, EFP03, ZSR02, and in a Bayesian framework [CJ95, GJ95]. (We will comment on this in more detail later.)

The complexity of a sensor selection problem (though not the one we consider) is considered in BKG06, where the authors show that it is NP-hard. (As far as we know, NPhardness of the sensor selection problem we consider has not been established.) The sensor selection problem can be exactly solved using global optimization techniques, such as branch and bound [Wel82, LW66]. These methods can, and often do, run for very long times, even with modest values of $k$ and $m$.

Several heuristics have been proposed to approximately solve the sensor selection problem. These include genetic algorithms [YSK93, and application specific local search methods. Local optimization techniques, similar to the one we describe, are summarized in [NM92, JD75]. While these heuristics can produce good suboptimal sensor selections, they do not yield any guarantees or bounds on the performance that is achievable. In any case, any local optimization method, including the ones described in these papers, and generic methods such as randomized rounding [MR95, can be incorporated into our method.

The sensor selection problem is closely related to the D-optimal experiment design problem Puk06, Fed72]. Here too we are to choose a subset of possible measurements from a pallette of choices. In D-optimal experiment design, however, we consider the case when $k$ 
and $m$ both grow, with a constant ratio; the question is not which sensors to use, but how frequently to use each one. The standard convex relaxation for the D-optimal experiment design problem (see, e.g., BV04, §7.5]) leads to a convex problem that is similar to ours, but different; we will discuss the differences in more detail below.

Finally, we note that the idea of using convex relaxation as the basis for a heuristic for solving a combinatorial problem is quite old, and has been observed to give very good results in many applications. Recent problems that are solved using this general technique are compressed sensing [Don06], sparse regressor selection [Tib96, sparse signal detection Tro06], sparse decoding [FKW03, and many others. Other applications that use convex relaxations include portfolio optimization with transaction costs [LFB07], controller design [HHB98, and circuit design VBG97.

Outline. The rest of this paper is organized as follows. In \$2 we formally describe the sensor selection problem. In $\$ 3$ we describe the basic convex relaxation, an approximate relaxation that can be solved even more efficiently, and a local optimization method to improve the basic sensor selection. We illustrate the method, with and without local optimization, with a numerical example, in $\$ 4$. In $\$ 5$ we describe a number of variations and extensions on the sensor selection problem, than can be incorporated in the convex optimization framework, including different objective functions, MAP estimation, constraints on sensors, and a robust version of the sensor selection problem.

\section{Sensor selection}

\subsection{Parameter estimation}

Suppose we are to estimate a vector $x \in \mathbf{R}^{n}$ from $m$ linear measurements, corrupted by additive noise,

$$
y_{i}=a_{i}^{T} x+v_{i}, \quad i=1, \ldots, m,
$$

where $x \in \mathbf{R}^{n}$ is a vector of parameters to estimate, and $v_{1}, \ldots, v_{m}$ are independent identically distributed $\mathcal{N}\left(0, \sigma^{2}\right)$ random variables. We assume that $a_{1}, \ldots, a_{m}$, which characterize the measurements, span $\mathbf{R}^{n}$. The maximum likelihood estimate of $x$ is

$$
\hat{x}=\left(\sum_{i=1}^{m} a_{i} a_{i}^{T}\right)^{-1} \sum_{i=1}^{m} y_{i} a_{i} .
$$

The estimation error $x-\hat{x}$ has zero mean and covariance

$$
\Sigma=\sigma^{2}\left(\sum_{i=1}^{m} a_{i} a_{i}^{T}\right)^{-1} .
$$

The $\eta$-confidence ellipsoid for $x-\hat{x}$, which is the minimum volume ellipsoid that contains $x-\hat{x}$ with probability $\eta$, is given by

$$
\mathcal{E}_{\alpha}=\left\{z \mid z^{T} \Sigma^{-1} z \leq \alpha\right\},
$$


where $\alpha=F_{\chi_{n}^{2}}^{-1}(\eta) . \quad\left(F_{\chi_{n}^{2}}\right.$ is the cumulative distribution function of a $\chi$-squared random variable with $n$ degrees of freedom.) A scalar measure of the quality of estimation is the volume of the $\eta$-confidence ellipsoid,

$$
\operatorname{vol}\left(\mathcal{E}_{\alpha}\right)=\frac{(\alpha \pi)^{n / 2}}{\Gamma(n / 2+1)} \operatorname{det} \Sigma^{1 / 2},
$$

where $\Gamma$ is the Gamma function. Another scalar measure of uncertainty, that has the same units as the entries in the parameter $x$, is the mean radius, defined as the geometric mean of the lengths of the semi-axes of the $\eta$-confidence ellipsoid,

$$
\rho\left(\mathcal{E}_{\alpha}\right)=\sqrt{\alpha}(\operatorname{det} \Sigma)^{1 / 2 n}
$$

We will be interested in volume ratios, so it is convenient to work with the log of the volume,

$$
\log \operatorname{vol}\left(\mathcal{E}_{\alpha}\right)=\beta-(1 / 2) \log \operatorname{det}\left(\sum_{i=1}^{m} a_{i} a_{i}^{T}\right),
$$

where $\beta$ is a constant that depends only on $\sigma, n$, and $\eta$. The log volume of the confidence ellipsoid, given in (6) , gives a quantitative measure of how informative the collection of $m$ measurements is.

\subsection{Sensor selection problem}

Now we can describe the sensor selection problem. We consider a set of $m$ potential measurements, characterized by $a_{1}, \ldots, a_{m} \in \mathbf{R}^{n}$; we are to choose a subset of $k(\geq n)$ of them that minimizes the log volume (or mean radius) of the resulting confidence ellipsoid. This can be expressed as the optimization problem

$$
\begin{array}{ll}
\operatorname{maximize} & \log \operatorname{det}\left(\sum_{i \in \mathcal{S}} a_{i} a_{i}^{T}\right) \\
\text { subject to } & |\mathcal{S}|=k
\end{array}
$$

where $S \subseteq\{1, \ldots, m\}$ is the optimization variable, and $|\mathcal{S}|$ denotes the cardinality of $\mathcal{S}$. (We interpret $\log \operatorname{det}\left(\sum_{i \in \mathcal{S}} a_{i} a_{i}^{T}\right)$ as $-\infty$ if $\sum_{i \in \mathcal{S}} a_{i} a_{i}^{T}$ is singular.) We let $p^{\star}$ denote the optimal value of the sensor selection problem.

We can rewrite the problem (17) as

$$
\begin{array}{ll}
\operatorname{maximize} & \log \operatorname{det}\left(\sum_{i=1}^{m} z_{i} a_{i} a_{i}^{T}\right) \\
\text { subject to } & \mathbf{1}^{T} z=k \\
& z_{i} \in\{0,1\}, \quad i=1, \ldots, m
\end{array}
$$

with variable $z \in \mathbf{R}^{m}$. (The vector $\mathbf{1}$ is the vector with all entries one.) Here $z_{i}$ encodes whether the $i$ th measurement (or sensor) is to be used. This problem is a Boolean-convex problem, since the objective is a concave function of $z$ for $z_{i} \geq 0$ (see, e.g., [BV04, §3.1.5]), the sum constraint is linear, and the last $m$ constraints restrict $z$ to be Boolean (i.e., 0-1). 


\section{Convex relaxation}

\subsection{The relaxed sensor selection problem}

By replacing the nonconvex constraints $z_{i} \in\{0,1\}$ with the convex constraints $z_{i} \in[0,1]$, we obtain the convex relaxation of the sensor selection problem (7):

$$
\begin{array}{ll}
\operatorname{maximize} & \log \operatorname{det}\left(\sum_{i=1}^{m} z_{i} a_{i} a_{i}^{T}\right) \\
\text { subject to } & \mathbf{1}^{T} z=k \\
& 0 \leq z_{i} \leq 1, \quad i=1, \ldots, m
\end{array}
$$

where $z \in \mathbf{R}^{m}$ is the variable. This problem, unlike the original sensor selection problem (17), is convex, since the objective (to be maximized) is concave, and the equality and inequality constraints on $z$ are linear. It can be solved efficiently, for example, using interior-point methods BV04. These methods typically require a few tens of iterations; each iteration can be carried out (as we will see below) with a complexity of $O\left(\mathrm{~m}^{3}\right)$ operations, so the overall complexity is $O\left(\mathrm{~m}^{3}\right)$ operations. We will let $z^{\star}$ denote a solution of the relaxed problem (9))

The relaxed sensor selection problem (9) is not equivalent to the original sensor selection problem (77); in particular, $z_{i}^{\star}$ can be fractional. We can say, however, that the optimal objective value of the relaxed sensor selection problem (9), which we denote $U$, is an upper bound on $p^{\star}$, the optimal objective value of the sensor selection problem (8). To see this, we note that the feasible set for the relaxed problem contains the feasible set for the original problem; therefore, its optimal value cannot be smaller than that of the original problem.

We can also use the solution $z^{\star}$ of the relaxed problem (9) to generate a suboptimal subset selection $\hat{\mathcal{S}}$. There are many ways to do this; but we describe here the simplest possible method. Let $z_{i_{1}}^{\star}, \ldots, z_{i_{m}}^{\star}$ denote the elements of $z^{\star}$ re-arranged in descending order. (Ties can be broken arbitrarily.) Our selection is then

$$
\hat{\mathcal{S}}=\left\{i_{1}, \ldots, i_{k}\right\},
$$

i.e., the indices corresponding to the $k$ largest elements of $z^{\star}$. We let $\hat{z}$ be the corresponding $0-1$ vector. The point $\hat{z}$ is feasible for the sensor selection problem (8); the associated objective value,

$$
L=\log \operatorname{det}\left(\sum_{i=1}^{m} \hat{z}_{i} a_{i} a_{i}^{T}\right),
$$

is then a lower bound on $p^{\star}$, the optimal value of the sensor selection problem (8)].

The difference between the upper and lower bounds on $p^{\star}$,

$$
\delta=U-L=\log \operatorname{det}\left(\sum_{i=1}^{m} z_{i}^{\star} a_{i} a_{i}^{T}\right)-\log \operatorname{det}\left(\sum_{i=1}^{m} \hat{z}_{i} a_{i} a_{i}^{T}\right),
$$

is called the gap. The gap is always nonnegative; if it is zero, then $\hat{z}$ is actually optimal for the sensor selection problem (88); more generally, we can say that the subset selection $\hat{z}$ is no more than $\delta$-suboptimal. 
We can relate the gap $\delta$, which is a difference of log-determinants, to geometric properties of confidence ellipsoids. A gap of $\delta$ corresponds to a ratio of $\exp (\delta / 2)$ in confidence ellipsoid volume. In terms of the mean radius $\rho$, a gap of $\delta$ corresponds to a ratio $\exp (\delta / 2 n)$.

Not much can be said about the gap, in general; for example, there are no generic useful bounds on how large it can be. The gap is, however, very useful when evaluated for a given problem instance.

\subsection{Relation to D-optimal experiment design}

Our sensor selection problem, and relaxed sensor selection problem, are closely related to D-optimal experiment design. In D-optimal experiment design, we have a set of potential measurements or sensors. In this case, however, we can use any one sensor multiple times; the problem is to choose which sensors to use, and for each one, how many times to use it, while keeping the total number of uses less than or equal to $k$. In contrast, in our sensor selection problem, we can use each potential sensor at most once. One method for approximately solving the D-optimal experiment design problem is to form a convex relaxation, that is the very similar to ours; however, the upper bound constraints $z_{i} \leq 1$ are not present, and the relaxed variables are normalized to have sum one (and not $k$ ); see, e.g., [BV04, §7.5]. The variables in the relaxed D-optimal experiment design problem also have a different interpretation: $z_{i}$ is the frequency with which sensor $i$ is to be used, when a large number of measurements is made.

\subsection{The dual problem}

In this section we describe a dual for the relaxed sensor selection problem, which has an interesting interpretation in terms of covering ellipsoids. The dual of the relaxed sensor selection problem is

$$
\begin{array}{ll}
\operatorname{minimize} & \log \operatorname{det} \Lambda^{-1}-n+k \nu+\mathbf{1}^{T} \mu \\
\text { subject to } & \nu+\mu_{i} \geq a_{i}^{T} \Lambda a_{i}, \quad i=1, \ldots, m \\
& \mu_{i} \geq 0, \quad i=1, \ldots, m
\end{array}
$$

with variables $\Lambda \in \mathbf{S}^{n}, \mu \in \mathbf{R}^{n}$, and $\nu \in \mathbf{R}$. (The set of $n \times n$ symmetric matrices is denoted by $\mathbf{S}^{n}$.) See Appendix $\mathrm{A}$ for the derivation.

This dual problem can be interpreted as the problem of finding the minimum volume covering ellipsoid with outlier detection; see [SF04, DPWH04]. (This should not be surprising because the dual of the D-optimal experiment design problem is the minimum volume covering ellipsoid problem [BV04, 7.5.3].)

If $\mu_{i}$ are set to 0 , the optimal solution $\nu^{*}$ and $\Lambda^{*}$ determine the minimum volume ellipsoid, given by $\left\{x \mid x^{T} \Lambda^{*} x \leq \nu^{*}\right\}$, that contains the $m$ points $a_{1}, \ldots, a_{m}$. When the variable $\mu_{i}$ is positive, $a_{i}$ is allowed to be outside this ellipsoid, i.e., it is an outlier. We now show that, at optimality, at most $k$ of the $\mu_{i}$ are nonzero. This can be inferred in many ways. Let $\Lambda^{\star}$, $\mu^{\star}$, and $\nu^{\star}$ be an optimal solution of the problem (10). The dual variables $\mu_{i}$ are associated 
with the inequalities $z_{i} \leq 1$ and by complementary slackness we have

$$
\mu_{i}^{\star}\left(1-z_{1}^{\star}\right)=0, \quad i=1, \ldots, m \text {. }
$$

Since all the $z_{i}^{\star}$ are positive and sum to $k$, at most $k$ of the $z_{i}^{\star}$ are 1 and thus at most $k$ of the $\mu_{i}^{\star}$ are nonzero. Therefore the solution of the problem (10) determines a covering ellipsoid for the $m$ points $a_{1}, \ldots, a_{m}$, with at most $k$ outliers.

\subsection{Approximate relaxed sensor selection}

It is not necessary to solve the relaxed sensor selection problem (9) to high accuracy, since we use it only to get the upper bound $U$, and to find the indices associated with the largest $k$ values of its solution. In this section we describe a simple method for solving it approximately but very efficiently, while retaining a provable upper bound on $p^{\star}$. This can be done by solving a smooth convex problem, which is closely related to the subproblems solved in an interior-point method for solving the relaxed problem.

The approximate relaxed sensor selection problem is

$$
\begin{array}{ll}
\operatorname{maximize} & \psi(z)=\log \operatorname{det}\left(\sum_{i=1}^{m} z_{i} a_{i} a_{i}^{T}\right)+\kappa \sum_{i=1}^{m}\left(\log \left(z_{i}\right)+\log \left(1-z_{i}\right)\right) \\
\text { subject to } & \mathbf{1}^{T} z=k,
\end{array}
$$

with variable $z \in \mathbf{R}^{m}$. Here $\kappa$ is a positive parameter that controls the quality of approximation. In the approximate relaxed sensor selection problem, we have implicit constraints that $z_{i} \in(0,1)$. The function $\psi$ is concave and smooth, so the problem (11) can be efficiently solved by Newton's method, which we describe in detail below. Let $z^{*}$ denote the solution of the approximate relaxed sensor selection problem (11).

A standard result in interior-point methods [BV04, $§ 11.2]$ is that $z^{*}$ is at most $2 m \kappa$ suboptimal for the relaxed sensor selection problem (9):

$$
\log \operatorname{det}\left(\sum_{i=1}^{m} z_{i}^{*} a_{i} a_{i}^{T}\right) \geq \log \operatorname{det}\left(\sum_{i=1}^{m} z_{i}^{\star} a_{i} a_{i}^{T}\right)-2 m \kappa=U-2 m \kappa .
$$

In particular, we can use

$$
\tilde{U}=\log \operatorname{det}\left(\sum_{i=1}^{m} z_{i}^{*} a_{i} a_{i}^{T}\right)+2 m \kappa
$$

as an upper bound on $p^{\star}$.

We can use this bound to choose $\kappa$ so that, in terms of $\rho$, the increase in gap contributed by the term $2 m \kappa$, which is a factor of $\exp (2 m \kappa / 2 n)$, is small, say, $1 \%$. This corresponds to $\kappa \approx 0.01 n / m$.

Newton's method. We now briefly describe Newton's method for solving (11); for full details see, e.g., BV04, §10.2]. As initial (feasible) point we take $z=(k / m) 1$. At each step, we compute the Newton search step $\Delta z_{\mathrm{nt}}$, which can be expressed as

$$
\Delta z_{\mathrm{nt}}=-\left(\nabla^{2} \psi\right)^{-1} \nabla \psi+\left(\frac{\mathbf{1}^{T}\left(\nabla^{2} \psi\right)^{-1} \nabla \psi}{\mathbf{1}^{T}\left(\nabla^{2} \psi\right)^{-1} \mathbf{1}}\right)\left(\nabla^{2} \psi\right)^{-1} \mathbf{1}
$$


We then use a backtracking line search to compute a step size $s \in(0,1]$, and update $z$ by replacing it with $z+s \Delta z_{\mathrm{nt}}$. We stop when the Newton decrement $\left(-\nabla \psi^{T} \Delta z_{\mathrm{nt}}\right)^{1 / 2}$ is small. The total number of steps required is typically ten or fewer.

For completeness we give expressions for the derivatives of $\psi$. Its gradient is given by

$$
(\nabla \psi)_{i}=a_{i}^{T} W a_{i}+\kappa / z_{i}-\kappa /\left(1-z_{i}\right), \quad i=1, \ldots, m
$$

where

$$
W=\left(\sum_{i=1}^{m} z_{i} a_{i} a_{i}^{T}\right)^{-1}
$$

The Hessian $\nabla^{2} \psi$ is given by

$$
\nabla^{2} \psi=-\left(A W A^{T}\right) \circ\left(A W A^{T}\right)-\kappa \operatorname{diag}\left(1 / z_{1}^{2}+1 /\left(1-z_{1}\right)^{2}, \ldots, 1 / z_{m}^{2}+1 /\left(1-z_{m}\right)^{2}\right),
$$

where $\circ$ denotes the Hadamard (elementwise) product and $A$ is the measurement matrix:

$$
A=\left[\begin{array}{c}
a_{1}^{T} \\
\vdots \\
a_{m}^{T}
\end{array}\right] .
$$

We can give a complexity analysis for computing the Newton step $\Delta z_{\text {nt }}$ using (13). We first form $\sum_{i=1}^{m} z_{i} a_{i} a_{i}^{T}$, which costs $O\left(m n^{2}\right)$ operations, and compute its Cholesky factor, which costs $O\left(n^{3}\right)$. We then form $A W A^{T}$ and $\nabla^{2} \psi$, which costs $O\left(m^{2} n\right)$. We compute its Cholesky factorization, which costs $O\left(\mathrm{~m}^{3}\right)$ (which dominates all other costs so far). Once we have computed the Cholesky factorization of $\nabla^{2} \psi$, we can compute $\Delta z_{\text {nt }}$ at cost $O\left(\mathrm{~m}^{2}\right)$. Thus, the overall cost is $O\left(\mathrm{~m}^{3}\right)$. Moreover, the hidden constant is quite modest, since the cost is dominated by the Cholesky factorization of an $m \times m$ matrix, which can be carried out in $(1 / 3) m^{3}$ operations.

\subsection{Local optimization}

The construction of a feasible selection $\hat{z}$ from the solution of the (approximate) relaxed problem (11) $z^{*}$ can be (possibly) improved by a local optimization method. One simple method to carry this out is to start from $\hat{z}$, and check sensor selections that can be derived from $\hat{z}$ by swapping one of the $k$ chosen sensors $\left(i . e ., i_{1}, \ldots, i_{k}\right)$ with one of the $m-k$ sensors not chosen. For similar methods, see, e.g., Fedorov's exchange algorithm [Fed72, MN94] or Wynn's algorithm Wyn72.

We can determine whether a sensor swap increases the objective value more efficiently than by computing the new objective value from scratch. Suppose we are to evaluate the change in objective value when sensor $j$ is removed from our selection, and sensor $l$ (which

was not originally chosen) replaces it. We let $\hat{\Sigma}$ denote the error covariance with the original subset selection,

$$
\hat{\Sigma}=\left(\sum_{i=1}^{m} \hat{z}_{i} a_{i} a_{i}^{T}\right)^{-1}
$$


and we let $\tilde{\Sigma}$ denote the error covariance when sensor $j$ is swapped with sensor $l$,

$$
\tilde{\Sigma}=\left(\sum_{i=1}^{m} \hat{z}_{i} a_{i} a_{i}^{T}-a_{j} a_{j}^{T}+a_{l} a_{l}^{T}\right)^{-1} .
$$

Using the low-rank update formula for the determinant of a matrix we have

$$
\begin{aligned}
\operatorname{det} \tilde{\Sigma}^{-1} & =\operatorname{det}\left(\hat{\Sigma}^{-1}-a_{j} a_{j}^{T}+a_{l} a_{l}\right) \\
& =\operatorname{det} \hat{\Sigma}^{-1} \operatorname{det}\left(I+\left[\begin{array}{c}
a_{j}^{T} \\
a_{l}^{T}
\end{array}\right] \hat{\Sigma}\left[\begin{array}{ll}
-a_{j} & a_{l}
\end{array}\right]\right) .
\end{aligned}
$$

We can determine whether swapping $j$ and $l$ increases the objective, i.e., whether $\operatorname{det} \tilde{\Sigma}^{-1}>$ $\operatorname{det} \hat{\Sigma}^{-1}$, by evaluating the determinant of the $2 \times 2$ matrix

$$
S=I+\left[\begin{array}{c}
a_{j}^{T} \\
a_{l}^{T}
\end{array}\right] \hat{\Sigma}\left[-a_{j} a_{l}\right] .
$$

The computation effort required to calculate this matrix is $O\left(n^{2}\right)$. (In contrast, computing det $\tilde{\Sigma}$ from scratch requires $O\left(n^{3}\right)$, so the savings here is $\left.O(n)\right)$. A small gain in efficiency can be obtained by recognizing

$$
\begin{aligned}
\operatorname{det} S & =1+a_{l}^{T} \hat{\Sigma} a_{l}-a_{j}^{T} \hat{\Sigma} a_{j}+\left(a_{l}^{T} \hat{\Sigma} a_{j}\right)^{2}-\left(a_{l}^{T} \hat{\Sigma} a_{l}\right)\left(a_{j}^{T} \hat{\Sigma} a_{j}\right) \\
& \leq 1+a_{l}^{T} \hat{\Sigma} a_{l}-a_{j}^{T} \hat{\Sigma} a_{j},
\end{aligned}
$$

remembering the previously calculated products of the form $a_{i}^{T} \hat{\Sigma} a_{i}$, and checking $a_{l}^{T} \hat{\Sigma} a_{l}>$ $a_{j}^{T} \hat{\Sigma} a_{j}$ before calculating $\operatorname{det} S$.

Now we continue our description of the local optimization method. Given the current sensor selection, we attempt a search over all possible $k(m-k)$ swaps. If we find that no swap increases the objective value, the algorithm terminates. The solution so obtained is called 2-opt, because exchanging any one selected sensor with any unselected one will not improve the solution.

If, however, we encounter a swap that increases the objective value, we (greedily) update $\hat{z}$ to correspond to the new sensor selection, replacing $\hat{\Sigma}$ by $\tilde{\Sigma}$. The matrix $\tilde{\Sigma}$ can be evaluated efficiently using the matrix inversion lemma (also known as Woodbury formula):

$$
\tilde{\Sigma}=\hat{\Sigma}-\hat{\Sigma}\left[\begin{array}{ll}
-a_{j} & a_{l}
\end{array}\right]\left(I+\left[\begin{array}{c}
a_{j}^{T} \\
a_{l}^{T}
\end{array}\right] \hat{\Sigma}\left[\begin{array}{ll}
-a_{j} & a_{l}
\end{array}\right]\right)^{-1}\left[\begin{array}{l}
a_{j}^{T} \\
a_{l}^{T}
\end{array}\right] \hat{\Sigma} .
$$

The computation effort required to calculate $\tilde{\Sigma}$ given $\hat{\Sigma}$ is $O\left(n^{2}\right)$. With the new sensor selection $\hat{z}$ we restart the search for an improving swap.

The local optimization algorithm must terminate because there is only a finite number of sensor selections that are better than the original one. The total number of local optimization steps can be very large (in theory); so we can simply limit the number of steps taken, say to $N_{\text {loc }}$. (We should mention that we have never observed an example that requires a very large number of local optimization steps.) If $N_{\text {loc }}$ is chosen to grow no faster than $\mathrm{m}^{3} / \mathrm{n}^{2}$, then the total computational effort of the local optimization method will be $O\left(\mathrm{~m}^{3}\right)$, the same as solving the relaxed sensor problem. 
More sophisticated local optimization. The local optimization method described above does not use the solution of the (approximate) relaxed sensor selection problem $z^{*}$; instead it proceeds directly from the rounded estimate $\hat{z}$. More sophisticated rounding methods can use the approximate relaxed point $z^{*}$. For example, in a randomized rounding scheme, $z_{i}^{*}$ is interpreted as the probability of selecting sensor $i$. In the local optimization method, we can use $z^{*}$ to order the sensors which are checked for possible swapping. (In the local optimization described above, the sensors are checked according to their index.) More specifically, we choose unselected sensors in descending order of the $z_{i}^{*}$ values, and we pick the selected sensors in ascending order of the $z_{i}^{*}$ values. The intuition behind this scheme is that a sensor with higher $z_{i}^{*}$ is more likely to be in the globally optimal sensor selection. To determine the ordering we need to sort the sensors according to the values only one time, and then maintain the ordering when a swap is taken. The initial sorting requires a computation effort of $O(m \log m)$, which for practical values of $m$ and $k$ is dominated by the computational effort needed to check the $k(m-k)$ swaps. We can also restrict the swaps to be among those sensors for which $z_{i}^{*}$ is in the interval $[0.1,0.9]$ (or some interval, possibly symmetric, around $1 / 2$ ). This drastically reduces the number of swaps to be checked (and number of sensors to be sorted), and therefore speeds up the local optimization.

\section{Example}

In this section we illustrate the sensor selection method with a numerical example. We consider an example instance with $m=100$ potential sensors and $n=20$ parameters to estimate. The measurement vectors $a_{1}, \ldots, a_{m}$ are chosen randomly, and independently, from an $\mathcal{N}(0, I / \sqrt{n})$ distribution. We solve the relaxed problem (111), with $\kappa=10^{-3}$, and find suboptimal subset selections, with and without local optimization, for $k=20,21, \ldots, 40$.

To solve each approximate relaxed problem requires 11 Newton steps, which would take a few milliseconds in a $\mathrm{C}$ implementation, run on a typical $2 \mathrm{GHz}$ personal computer. For each problem instance, the (basic) local search checks 4000-12000 sensor swaps, and around 3-20 swaps are taken before a 2-opt solution is found. We also the run the restricted version on the local search, which only considers sensors with $z_{i}^{*}$ value in the interval $[0.1,0.9]$. This local search produces an equally good final sensor selection, while checking a factor 10-15 times fewer swaps than the basic method. (In any case, the basic local search only takes milliseconds to complete, on a typical personal computer, for a problem instance of this size.)

To show the quality of the sensor subsets chosen, we evaluate the upper bound $\tilde{U}$ (given by (12)), the lower bound $L$ using the simple selection rule, and the (possibly) better lower bounds $L_{\mathrm{loc}}$ and $L_{\mathrm{loc}(\mathrm{r})}$ obtained after local optimization and restricted local optimization, respectively, for each value of $k$. The top half of Figure 1 shows $\tilde{U}, L, L_{\mathrm{loc}}$, and $L_{\mathrm{loc}(\mathrm{r})}$, and the bottom half shows the gaps $\delta=\tilde{U}-L, \delta_{\mathrm{loc}}=\tilde{U}-L_{\mathrm{loc}}$, and $\delta_{\mathrm{loc}(\mathrm{r})}=\tilde{U}-L_{\mathrm{loc}(\mathrm{r})}$. We also express the gaps as the ratio of mean radii, $\exp (\delta / 2 n), \exp \left(\delta_{\text {loc }} / 2 n\right)$ and $\exp \left(\delta_{\text {loc }(\mathrm{r})} / 2 n\right)$, in Figure 2 .

These plots show that very good sensor selections are obtained. For example, with $k=25$, the relaxation followed by 2 -opt local optimization produces a design which is at 

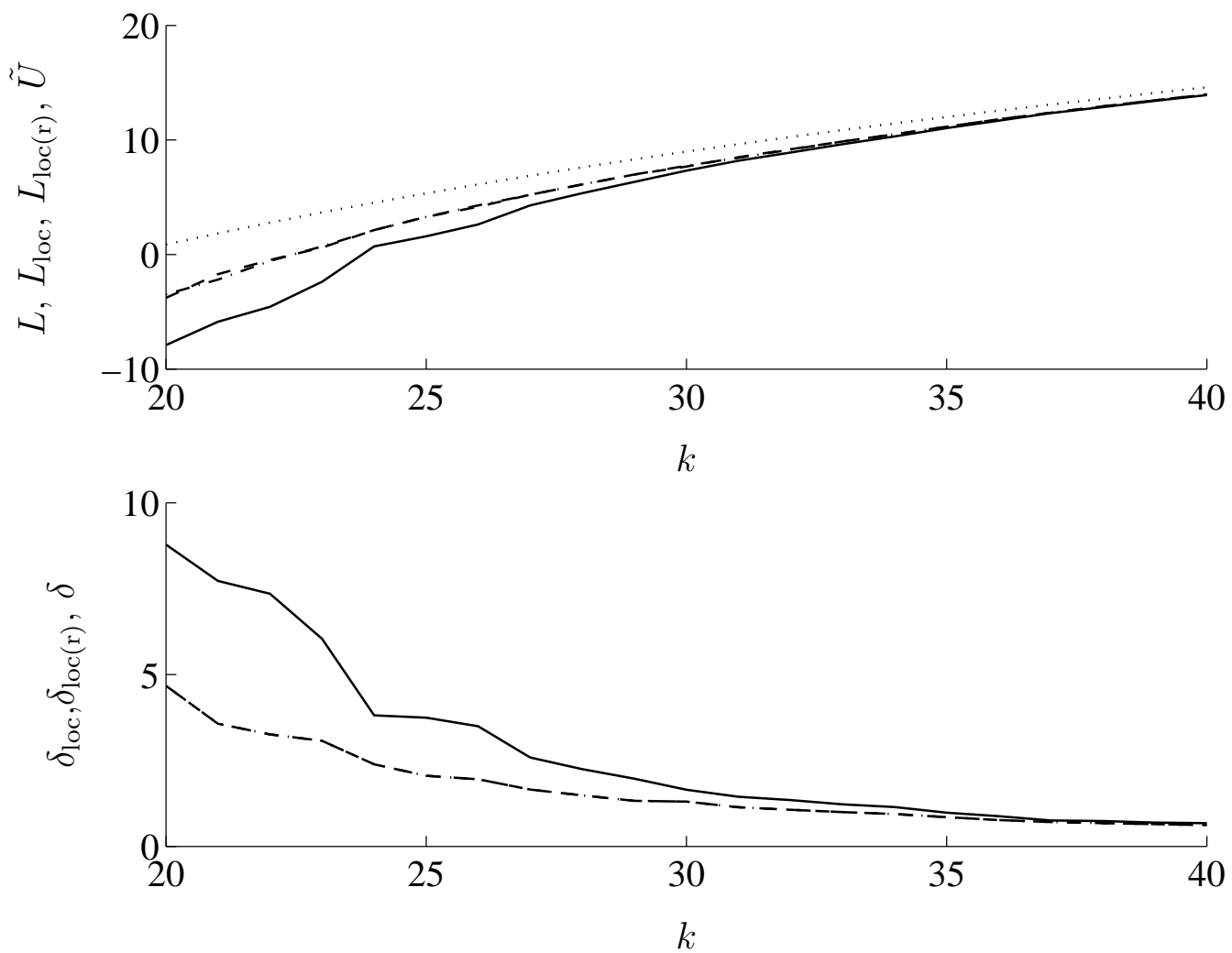

Figure 1: Top: Upper bound $\tilde{U}$ (top curve); lower bounds $L_{\mathrm{loc}}$ and $L_{\mathrm{loc}(\mathrm{r})}$ (middle curves); lower bound $L$ (bottom curve). Bottom: Gap $\delta$ (top curve); $\delta_{\mathrm{loc}}$ and $\delta_{\mathrm{loc}(\mathrm{r})}$ (bottom curves). 


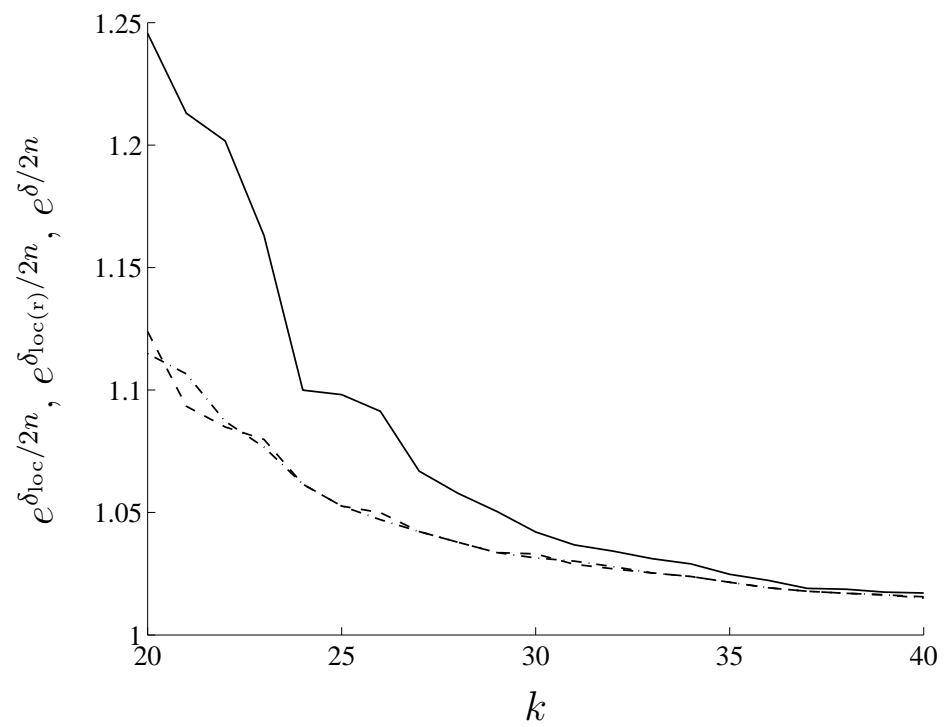

Figure 2: Gaps expressed as ratios of mean radii: $\exp (\delta / 2 n)$ (top curve); $\exp \left(\delta_{\mathrm{loc}} / 2 n\right)$ and $\exp \left(\delta_{\mathrm{loc}(\mathrm{r})} / 2 n\right)$ (bottom curves).

most $5.3 \%$ suboptimal, as measured by mean radius of the confidence ellipsoid. (This is only a bound; it is likely that the sensor selection found is closer to optimal than 5.3\%.) We can see that restricted local optimization performs as well as basic local optimization; the two curves are barely distinguishable. (In the figures, the values corresponding to the basic local optimization are shown by the dashed curve, and to the restricted local optimization are shown by the dash-dotted curve.) To find the globally optimally best sensor selection by direct enumeration would require evaluating the objective $\left(\begin{array}{c}100 \\ 25\end{array}\right)$ times, which is on the order of $10^{23}$ times, and clearly not practical.

\section{Extensions}

\subsection{Other measures of estimation quality}

So far we have used the volume of the confidence ellipsoid as our measure of the quality of estimation obtained by a sensor subset selection. Several other measures can be used instead of this one.

Mean squared error. The mean squared error in estimating the parameter $x$ is

$$
\mathbf{E}\left(\|x-\hat{x}\|^{2}\right)=\operatorname{tr}(\Sigma)
$$


The associated sensor selection problem can be expressed as the optimization problem

$$
\begin{array}{ll}
\operatorname{minimize} & \operatorname{tr}\left(\sum_{i=1}^{m} z_{i} a_{i} a_{i}^{T}\right)^{-1} \\
\text { subject to } & \mathbf{1}^{T} z=k \\
& z_{i} \in\{0,1\}, \quad i=1, \ldots, m
\end{array}
$$

with variable $z \in \mathbf{R}^{m}$. The optimization problem obtained by relaxing the 0 - 1 constraint is convex; see [BV04, §7.5]. (In the context of experiment design, this measure leads to so-called A-optimal experiment design.)

Worst case error variance. The variance of the estimation error in the direction $q \in \mathbf{R}^{n}$, with $\|q\|=1$, is

$$
\mathbf{E}\left(q^{T} x\right)^{2}=q^{T} \Sigma q .
$$

The worst case variance of the estimation error, over all directions, is

$$
\max _{\|q\|=1} q^{T} \Sigma q=\lambda_{\max }(\Sigma)
$$

the maximum eigenvalue of $\Sigma$. The associated sensor selection problem can be expressed as the optimization problem

$$
\begin{array}{ll}
\operatorname{maximize} & \lambda_{\min }\left(\sum_{i=1}^{m} z_{i} a_{i} a_{i}^{T}\right) \\
\text { subject to } & \mathbf{1}^{T} z=k \\
& z_{i} \in\{0,1\}, \quad i=1, \ldots, m,
\end{array}
$$

with variable $z \in \mathbf{R}^{m}$. Relaxing the $0-1$ constraint we obtain a convex problem. (In the context of experiment design, this measure leads to so-called E-optimal experiment design.)

Worst case coordinate error variance. The variance of the $i$ th coordinate of the estimation error, $x_{i}-\hat{x}_{i}$, is $\Sigma_{i i}$. The worst case coordinate error variance is the largest diagonal entry of the covariance matrix. Choosing the sensor subset to minimize this measure can be expressed as the problem

$$
\begin{array}{ll}
\operatorname{minimize} & \max _{j=1, \ldots, n}\left(\left(\sum_{i=1}^{m} z_{i} a_{i} a_{i}^{T}\right)^{-1}\right)_{j j} \\
\text { subject to } & \mathbf{1}^{T} z=k \\
& z_{i} \in\{0,1\}, \quad i=1, \ldots, m,
\end{array}
$$

with variable $z$. Relaxing the 0 -1 constraint we obtain a convex problem.

In fact the problem can be transformed to a semidefinite program (SDP), and therefore efficiently solved. Writing the problem (15) in epigraph form and relaxing the 0-1 constraint, we obtain

$$
\begin{array}{ll}
\operatorname{minimize} & t \\
\text { subject to } & t \geq e_{j}^{T}\left(\left(\sum_{i=1}^{m} z_{i} a_{i} a_{i}^{T}\right)^{-1}\right) e_{j}, \quad j=1, \ldots, m \\
& \mathbf{1}^{T} z=k \\
& 0 \leq z_{i} \leq 1, \quad i=1, \ldots, m,
\end{array}
$$


with variables $z \in \mathbf{R}^{m}$ and $t \in \mathbf{R}$. The vector $e_{i}$ is the vector with 1 in the $i$ th entry and 0 in the rest of the entries. This problem is equivalent to

$$
\begin{array}{ll}
\text { minimize } & t \\
\text { subject to } & {\left[\begin{array}{cc}
t & e_{j}^{T} \\
e_{j} & \sum_{i=1}^{m} z_{i} a_{i} a_{i}^{T}
\end{array}\right] \succeq 0, \quad j=1, \ldots, m} \\
& \mathbf{1}^{T} z=k \\
& 0 \leq z_{i} \leq 1, \quad i=1, \ldots, m,
\end{array}
$$

with variables $z$ and $t$. (The symbol $\succeq$ represents inequality with respect to the positive semidefinite matrix cone.) This is a semidefinite program.

\subsection{Sensor selection constraints}

Many constraints on the selection of the sensors can be represented as linear equalities or inequalities on the variable $z$, and so are easily incorporated into the convex relaxation. We describe some typical cases below.

\section{Logical constraints.}

- 'Only when' constraints. The constraint that sensor $i$ can be chosen only when sensor $j$ is also chosen can be expressed as $z_{i} \leq z_{j}$.

- 'Not both' constraints. The constraint that sensor $i$ and sensor $j$ cannot both be chosen can be expressed as $z_{i}+z_{j} \leq 1$.

- 'At least one of' constraints. To require that one of sensor $i$ or sensor $j$ be chosen, we impose the constraint $z_{i}+z_{j} \geq 1$.

These are easily extended to more complex situations. For example, to require that exactly two of the four sensors $i, j, k, l$ be chosen, we impose the linear equality constraint $z_{i}+z_{j}+$ $z_{k}+z_{l}=2$.

Budget constraints. In addition to limiting the number of sensors chosen to $k$, we can impose other resource limitations on the sensor selection. Suppose that $c_{i}$ is some cost (say, in dollars, power, or weight) associated with choosing sensor $i$. We can impose a budget constraint on the selection, i.e., a maximum allowed cost for the selection, as $c^{T} z \leq B$, where $B$ is the budget.

\subsection{Vector measurements}

In our setup so far, each sensor gives a scalar measurement. Now suppose the measurements $y_{i}$ are vectors, i.e., sensor $i$ gives not one, but several scalar measurements of the parameters. The $m$ potential measurements are

$$
y_{i}=A_{i}^{T} x+v_{i}, \quad i=1, \ldots, m,
$$


where $y_{i} \in \mathbf{R}^{m_{i}}, x \in \mathbf{R}^{n}$. The measurement noises $v_{1}, \ldots, v_{m}$ are independent random variables, with $\mathcal{N}\left(0, \sigma^{2} I\right)$ distribution. The sensor selection problem can be expressed as

$$
\begin{array}{ll}
\operatorname{maximize} & \log \operatorname{det}\left(\sum_{i=1}^{m} z_{i} A_{i} A_{i}^{T}\right) \\
\text { subject to } & \mathbf{1}^{T} z=k \\
& z_{i} \in\{0,1\}, \quad i=1, \ldots, m
\end{array}
$$

with variable $z \in \mathbf{R}^{m}$. Relaxing the $0-1$ constraint we obtain a convex problem. (The same problem can be also be obtained by associating each component of $y_{i}$ with a separate measurement, and adding constraints that require that if any scalar measurement from $y_{i}$ is used, all must be.)

\subsection{MAP estimation}

We have so far worked with maximum likelihood estimation. We can easily extend the method to the Bayesian framework. Suppose the prior density of $x$ is $\mathcal{N}\left(0, \Sigma_{x}\right)$. The maximum a posteriori probability (MAP) estimate of $x$, with selected sensors characterized by $z$, is

$$
\hat{x}_{\text {map }}=\left(\sigma^{-2} \sum_{i=1}^{m} z_{i} a_{i} a_{i}^{T}+\Sigma_{x}^{-1}\right)^{-1} \sum_{i=1}^{m} y_{i} a_{i} .
$$

The estimation error $x-\hat{x}_{\text {map }}$ has zero mean and covariance

$$
\Sigma_{\text {map }}(z)=\left(\sigma^{-2} \sum_{i=1}^{m} z_{i} a_{i} a_{i}^{T}+\Sigma_{x}^{-1}\right)^{-1} .
$$

The problem of choosing $k$ sensors to minimize the volume of the resulting $\eta$-confidence ellipsoid reduces to

$$
\begin{array}{ll}
\operatorname{maximize} & \log \operatorname{det}\left(\sigma^{-2} \sum_{i=1}^{m} z_{i} a_{i} a_{i}^{T}+\Sigma_{x}^{-1}\right) \\
\text { subject to } & \mathbf{1}^{T} z=k \\
& z_{i} \in\{0,1\}, \quad i=1, \ldots, m
\end{array}
$$

with variable $z \in \mathbf{R}^{m}$. Relaxing the 0-1 constraint results in a convex optimization problem.

Since the log det of the covariance matrix of a Gaussian random variable is the entropy of the random variable (differing by a constant), the problem (17) can be obtained via an information theoretic approach. Let $\tilde{y}(z)$ be the sensor measurement vector when sensors characterized by $z$ are chosen. The problem of choosing $k$ sensors to minimize the entropy of the random variable $x \mid \tilde{y}(z)$, or to maximize the mutual information between $x$ and the resulting measurement vector $\tilde{y}(z)$, is the problem (17).

\subsection{Estimating a linear function of the parameters}

Suppose the goal is to estimate $\tilde{x}=C x \in \mathbf{R}^{\tilde{n}}$, a linear function of the parameter $x$, where $C \in \mathbf{R}^{\tilde{n} \times n}$ has rank $\tilde{n}$. The prior density of $x$ is $\mathcal{N}\left(0, \Sigma_{x}\right)$, so the prior density of $\tilde{x}$ is 
$\mathcal{N}\left(0, \Sigma_{\tilde{x}}\right)$, where $\Sigma_{\tilde{x}}=C \Sigma_{x} C^{T}$. The covariance of the error of the MAP estimate of $\tilde{x}$ is $C \Sigma_{\text {map }}(z) C^{T}$, where $\Sigma_{\text {map }}(z)$ is given by (16). The problem of choosing $k$ sensors to minimize the volume of the resulting confidence ellipsoid is

$$
\begin{array}{ll}
\operatorname{minimize} & \log \operatorname{det}\left(C\left(\sigma^{-2} \sum_{i=1}^{m} z_{i} a_{i} a_{i}^{T}+\Sigma_{\tilde{x}}^{-1}\right)^{-1} C^{T}\right) \\
\text { subject to } & \mathbf{1}^{T} z=k \\
& z_{i} \in\{0,1\}, \quad i=1, \ldots, m
\end{array}
$$

with variable $z \in \mathbf{R}^{m}$.

Relaxing the constraints $z_{i} \in\{0,1\}$ to $0 \leq z_{i} \leq 1$ yields a convex problem. This relaxed problem can be solved directly by the Newton's method described in $\$ 3.4$, for which we need the gradient and Hessian of the objective function. The objective function is

$$
\phi(z)=\log \operatorname{det}\left(C X C^{T}\right)
$$

where $X \in \mathbf{S}^{n}$ is

$$
X=\left(\sigma^{-2} \sum_{i=1}^{m} z_{i} a_{i} a_{i}^{T}+\Sigma_{\tilde{x}}^{-1}\right)^{-1} .
$$

(To simplify notation we do not write $X$ explicitly as a function of $z$.) The gradient of the function $\phi$ is

$$
(\nabla \phi)_{i}=-\sigma^{-2} a_{i}^{T} X C^{T}\left(C X C^{T}\right)^{-1} C X a_{i}, \quad i=1, \ldots, m .
$$

The Hessian of the function $\phi$ is

$$
\begin{array}{r}
\left(\nabla^{2} \phi\right)_{i j}=2 \sigma^{-4}\left(a_{i}^{T} X a_{j}\right)\left(a_{i}^{T} X C^{T}\left(C X C^{T}\right)^{-1} C X a_{j}\right)-\sigma^{-4}\left(a_{i}^{T} X C^{T}\left(C X C^{T}\right)^{-1} C X a_{j}\right)^{2}, \\
i=1, \ldots, m, \quad j=1, \ldots, m,
\end{array}
$$

which can be written compactly as

$$
\nabla^{2} \phi=\sigma^{-4}(2 P-Q) \circ Q
$$

where

$$
P=A X A^{T}, \quad Q=A X C^{T}\left(C X C^{T}\right)^{-1} C X A^{T},
$$

and $A$ is given by (14).

The problem (18) can also be solved by transforming it to a standard one. We introduce a new variable, a lower triangular matrix $L \in \mathbf{R}^{\tilde{n} \times \tilde{n}}$, and write the relaxed version of the problem (18) as

$$
\begin{array}{ll}
\operatorname{minimize} & \log \operatorname{det}\left(L L^{T}\right)^{-1} \\
\text { subject to } & \mathbf{1}^{T} z=k \\
& 0 \leq z_{i} \leq 1, \quad i=1, \ldots, m \\
& \left(L L^{T}\right)^{-1} \succeq C\left(\sigma^{-2} \sum_{i=1}^{m} z_{i} a_{i} a_{i}^{T}+\Sigma_{\tilde{x}}^{-1}\right)^{-1} C^{T} \\
& L \text { lower triangular, }
\end{array}
$$


with variables $z \in \mathbf{R}^{m}$ and $L \in \mathbf{R}^{\tilde{n} \times \tilde{n}}$. The objective function is

$$
\log \operatorname{det}\left(L L^{T}\right)^{-1}=-2 \log \operatorname{det}(L)=-2 \sum_{i=1}^{\tilde{n}} \log L_{i i}
$$

since $L$ is lower triangular. The constraint that the lower triangular matrix $L$ is invertible is implicit, since the objective function requires that $L_{i i}>0$.

The matrix inequality

$$
\left(L^{T}\right)^{-1} L^{-1} \succeq C\left(\sigma^{-2} \sum_{i=1}^{m} z_{i} a_{i} a_{i}^{T} \Sigma_{\tilde{x}}^{-1}\right)^{-1} C^{T}
$$

can be written as

$$
I \succeq L^{T} C\left(\sigma^{-2} \sum_{i=1}^{m} z_{i} a_{i} a_{i}^{T}+\Sigma_{\tilde{x}}^{-1}\right)^{-1} C^{T} L,
$$

which is equivalent to

$$
\left[\begin{array}{cc}
I & L^{T} C \\
C^{T} L & \sigma^{-2} \sum_{i=1}^{m} z_{i} a_{i} a_{i}^{T}+\Sigma_{\tilde{x}}^{-1}
\end{array}\right] \succeq 0 .
$$

(Here we use $\sum_{i=1}^{m} z_{i} a_{i} a_{i}^{T}+\Sigma_{\tilde{x}}^{-1} \succ 0$.)

The problem (19) is therefore equivalent to

$$
\begin{array}{ll}
\operatorname{maximize} & \sum_{i=1}^{\tilde{n}} \log L_{i i} \\
\text { subject to } & \mathbf{1}^{T} z=k \\
& 0 \leq z_{i} \leq 1, \quad i=1, \ldots, m \\
& {\left[\begin{array}{cc}
I & L^{T} C \\
C^{T} L & \sigma^{-2} \sum_{i=1}^{m} z_{i} a_{i} a_{i}^{T}+\Sigma_{\tilde{x}}^{-1}
\end{array}\right] \succeq 0} \\
& L \text { lower triangular, }
\end{array}
$$

with variables $z \in \mathbf{R}^{m}$ and $L \in \mathbf{R}^{\tilde{n} \times \tilde{n}}$.

A similar approach can handle the problem of estimating a linear function of the variable $x$ in the maximum likelihood framework, but this requires additional technical conditions.

\subsection{Robust sensor selection}

In this section we consider the sensor problem with some uncertainty in the measurement vectors. The uncertainty is characterized by a given set $\mathcal{A}$ in which the measurement matrix $A$, given by (14), can take any value. In terms of $A$, the objective of the sensor selection problem (8) can be written as

$$
\log \operatorname{det}\left(A^{T} \operatorname{diag}(z) A\right),
$$

where $\operatorname{diag}(z) \in \mathbf{R}^{m \times m}$ is the diagonal matrix with entries $z_{1}, \ldots, z_{m}$. 
In the robust sensor selection problem we choose $k$ sensors to minimize the worst case mean radius of the resulting confidence ellipsoid, which can be written as

$$
\begin{array}{ll}
\operatorname{maximize} & \inf _{A \in \mathcal{A}} \log \operatorname{det}\left(A^{T} \operatorname{diag}(z) A\right) \\
\text { subject to } & \mathbf{1}^{T} z=k \\
& z_{i} \in\{0,1\}, \quad i=1, \ldots, m,
\end{array}
$$

with variables $z \in \mathbf{R}^{m}$. The problem data is the set $\mathcal{A}$.

The objective function of the robust optimization is problem (21), for $z_{i} \geq 0$, is the infimum of a family of concave functions, and therefore concave. Thus the problem (21), after relaxing the $0-1$ constraints, is a convex optimization problem.

The relaxed robust sensor selection problem can be written as

$$
\begin{array}{ll}
\text { maximize } & \log \operatorname{det}(U) \\
\text { subject to } & \mathbf{1}^{T} z=k \\
& 0 \leq z_{i} \leq 1, \quad i=1, \ldots, m \\
& U \preceq A^{T} \operatorname{diag}(z) A, \quad \text { for all } A \in \mathcal{A},
\end{array}
$$

with variables $z \in \mathbf{R}^{m}$ and the symmetric (positive definite) matrix $U \in \mathbf{R}^{m \times m}$. If the set $\mathcal{A}$ is finite, this is a standard convex optimization problem. If the set $\mathcal{A}$ is not finite, which usually is the case, the problem is a semi-infinite convex optimization problem, which can be solved using various general techniques, such as sampling; see, e.g., [HK93, MB07. In some cases (as we will see below), the semi-infinite problem can be simplified and solved.

We now consider the specific uncertainty model

$$
A \in \mathcal{A}=\left\{\bar{A}+\Delta \mid\|\Delta\|_{2} \leq \epsilon\right\}
$$

the semi-infinite constraint can represented as a (simple) linear matrix inequality (LMI) constraint, thereby simplifying the robust sensor selection problem to a standard SDP. The constraint $\|\Delta\|_{2} \leq \epsilon$ can be written as $\Delta^{T} \Delta \preceq \epsilon^{2} I$. The semi-infinite constraint in terms of $\bar{A}$ and $\Delta$ is

$$
U \preceq \bar{A}^{T} \operatorname{diag}(z) \bar{A}+\bar{A}^{T} \operatorname{diag}(z) \Delta+\Delta^{T} \operatorname{diag}(z) \bar{A}+\Delta^{T} \operatorname{diag}(z) \Delta, \quad \text { for all } \Delta^{T} \Delta \preceq \epsilon^{2} I .
$$

Theorem 3.3 in [LSZ04, §3] implies that the above semi-infinite quadratic matrix inequality holds if and only if the matrix inequality

$$
\left[\begin{array}{cc}
\bar{A}^{T} \operatorname{diag}(z) \bar{A}-U-t I & \bar{A}^{T} \operatorname{diag}(z) \\
\operatorname{diag}(z) \bar{A} & \operatorname{diag}(z)+\left(t / \epsilon^{2}\right) I
\end{array}\right] \succeq 0
$$

is feasible for some $t \geq 0$. The matrix inequality is linear in $z, U$, and $t$. The relaxed robust sensor selection for the uncertainty model (22) is

$$
\begin{array}{lll}
\text { maximize } & \log \operatorname{det}(U) \\
\text { subject to } & \mathbf{1}^{T} z=k \\
& 0 \leq z_{i} \leq 1, \quad i=1, \ldots, m \\
& {\left[\begin{array}{cc}
\bar{A}^{T} \operatorname{diag}(z) \bar{A}-U-t I & \bar{A}^{T} \operatorname{diag}(z) \\
\operatorname{diag}(z) \bar{A} & \operatorname{diag}(z)+\left(t / \epsilon^{2}\right) I
\end{array}\right] \succeq 0}
\end{array}
$$

with variables $z, U$, and $t$. 


\subsection{Example}

In this section we consider an example that combines three of the extensions. We consider a discrete-time linear dynamical system

$$
x(t+1)=F x(t), \quad t=1, \ldots, m+1,
$$

where $x(t) \in \mathbf{R}^{n}$ is the state at time $t$, and $F \in \mathbf{R}^{n \times n}$ is the dynamics matrix, which we assume is invertible. We have linear noise corrupted measurements,

$$
y(t)=H x(t)+v(t), \quad t=1, \ldots, m,
$$

where $y(t) \in \mathbf{R}^{p}$ is the measurement at time $t, v(t) \in \mathbf{R}^{p}$ is the measurement noise at time $t$, and $H \in \mathbf{R}^{p \times n}$ is the measurement matrix. We assume the noise vectors $v_{1}, \ldots, v_{m}$ are independent identically distributed $\mathcal{N}(0, I)$ random variables. The initial state $x(1)$ has a prior probability density $\mathcal{N}\left(0, \Sigma_{1}\right)$, and is independent of the noise vectors.

We consider the problem of choosing a set of $k$ (vector) measurements out of the $m$ (vector) measurements of the state, in order to minimize the mean squared error in estimating $x(m+1)$. This corresponds to choosing a set of $k$ times (out of the $m$ possible times) at which to obtain the measurements.

We can express the measurements as

$$
\left[\begin{array}{c}
y(1) \\
\vdots \\
y(m)
\end{array}\right]=\left[\begin{array}{c}
H F^{-m} \\
\vdots \\
H F^{-1}
\end{array}\right] x(m+1)+\left[\begin{array}{c}
v(1) \\
\vdots \\
v(m)
\end{array}\right] .
$$

The prior density of $x(m+1)$ is $\mathcal{N}\left(0, \Sigma_{m+1}\right)$, where $\Sigma_{m+1}=F^{m} \Sigma_{1} F^{m T}$. The MAP estimation error for $x(m+1)$ is zero mean, with covariance

$$
\Sigma(z)=\left(\sum_{i=1}^{m} z_{i}\left(H F^{-m+i-1}\right)^{T} H F^{-m+i-1}+\Sigma_{m+1}^{-1}\right)^{-1},
$$

where $z \in \mathbf{R}^{m}$ characterizes the selected measurement times. The problem of choosing $k$ times at which to take state measurements, in order to minimize the resulting mean square estimation error of $x(m+1)$, can be formulated as

$$
\begin{array}{ll}
\operatorname{minimize} & \operatorname{tr}(\Sigma(z)) \\
\text { subject to } & \mathbf{1}^{T} z=k \\
& z \in\{0,1\}, \quad i=1, \ldots, m,
\end{array}
$$

where $z$ is variable. Relaxing the $0-1$ constraints we obtain a convex optimization problem, which can be transformed to the semidefinite program

$$
\begin{array}{ll}
\operatorname{minimize} & \operatorname{tr}(Y) \\
\text { subject to } & \mathbf{1}^{T} z=k \\
& 0 \leq z_{i} \leq 1, \quad i=1, \ldots, m \\
& {\left[\begin{array}{cc}
Y & I \\
I & \sum_{i=1}^{m} z_{i}\left(H F^{-m+i-1}\right)^{T} H F^{-m+i-1}+\Sigma_{m+1}^{-1}
\end{array}\right] \succeq 0}
\end{array}
$$




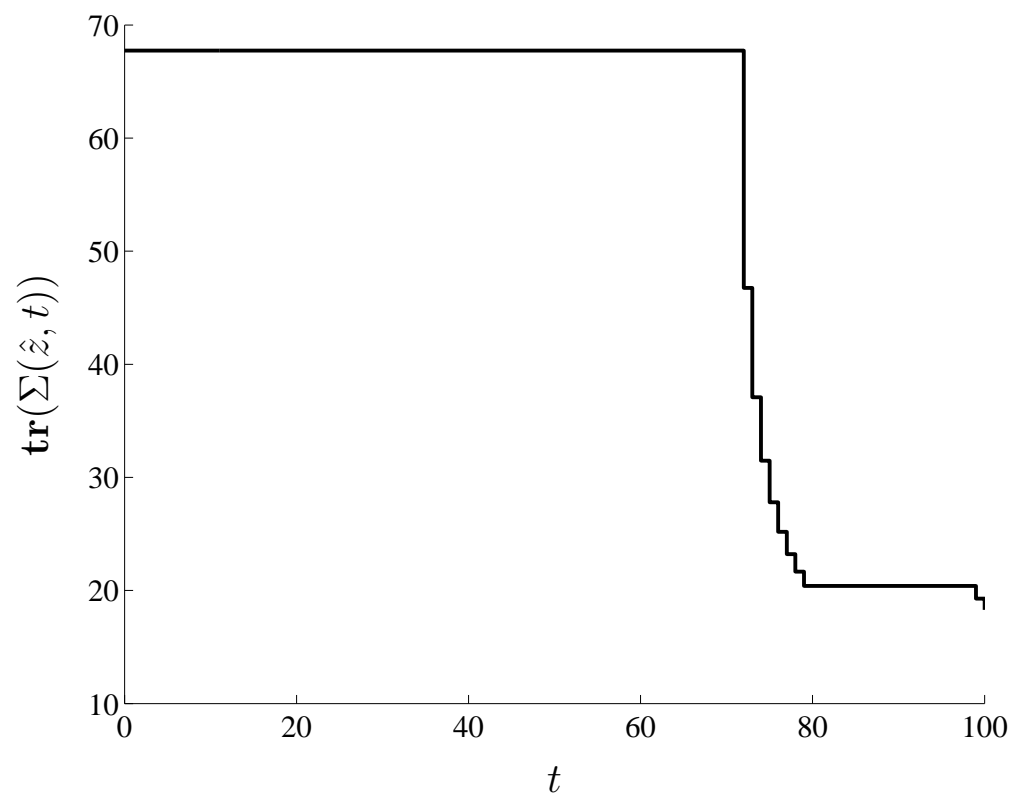

Figure 3: Mean squared error versus time for the sample time selection characterized by $\hat{z}$.

with variables $z \in \mathbf{R}^{m}$ and $Y \in \mathbf{S}^{n}$.

We now consider a numerical instance of the problem. We take state dimension $n=5$, measurement dimension $p=2$, over time interval $m=100$, out of which we are to choose $k=10$ times. We take the covariance of $x(1)$ to be $\Sigma_{1}=I$. The dynamics matrix $F$ has eigenvalues

$$
0.99 \pm 0.05 i, \quad 1.01 \pm 0.03 i, \quad 0.98
$$

i.e., a slowing growing, and a slowing decaying, oscillatory modes. The entries of the matrix $H$ are chosen independently from the uniform distribution on $[-0.1,0.1]$.

We solve the semidefinite program (26) using CVX GBY07 to obtain the solution of the relaxed problem $z^{\star}$, and select the $k$ times with the largest values of $z_{i}^{\star}$. The chosen times are

$$
72, \ldots, 79, \quad 99, \quad 100 .
$$

The objective value (mean square error) for this choice of 10 sample times is very close to the lower bound, given by the optimal value of the problem (25), so our choice is near globally optimal (and in particular, there is no need for local optimization).

In Figure 3 we plot the mean square estimation error of $x(m+1)$, given the chosen measurements up to time $t$, which is given by $\operatorname{tr}(\Sigma(\hat{z}, t))$, where

$$
\Sigma(\hat{z}, t)=\left(\sum_{i=1}^{t} \hat{z}_{i}\left(H F^{-m+i-1}\right)^{T} H F^{-m+i-1}+\Sigma_{m+1}^{-1}\right)^{-1}, \quad t=0, \ldots, m,
$$

where $\hat{z}$ is the (0-1) sample time selection. This mean square error drops after each measurement is taken. We can see that the largest drop in mean square estimation error occurs 
during the burst of measurements taken over the interval $t=72$ to $t=79$; a small further improvement occurs in the last two time steps.

\section{Conclusion}

The problem of choosing $k$ sensors or measurements, from among a set of candidate measurements, in order to obtain the best resulting estimate of some parameters, is in general a difficult combinatorial problem. We have shown, however, that convex relaxation, followed by a local optimization method, can often work very well. In particular, this method produces not only a suboptimal choice of measurements, but also, a bound on how well the globally optimal choice does. The performance achieved by the suboptimal choice is often very close to the global bound, which certifies that the choice is nearly optimal. Our method does not give a prior guarantee on this gap; but each time the method is used, on a particular problem instance, we get a specific bound.

\section{Acknowledgments}

We thank the anonymous reviewers for their helpful comments, Bruno Sinopoli for suggesting the problem to us, and Danny Bickson of the Hebrew University of Jerusalem for the initial suggestion that we analyze the dual of the sensor selection problem.

This material is based upon work supported by the Stanford Graduate Fellowship, by the Focus Center Research Program Center for Circuit \& System Solutions award 2003CT-888, by JPL award I291856, by the Precourt Institute on Energy Efficiency, by Army award W911NF-07-1-0029, by NSF award ECS-0423905, by NSF award 0529426, by DARPA award N66001-06-C-2021, by NASA award NNX07AEIIA, by AFOSR award FA9550-06-10514, and by AFOSR award FA9550-06-1-0312.

\section{Appendix}

\section{A Derivation of the dual problem}

In this section we derive the dual of the relaxed sensor selection problem (9). We introduce a new variable $X=\sum_{i=1}^{m} z_{i} a_{i} a_{i}^{T}$ and write the relaxed sensor selection problem (9) as

$$
\begin{array}{ll}
\operatorname{minimize} & \log \operatorname{det} X^{-1} \\
\text { subject to } & X=\sum_{i=1}^{m} z_{i} a_{i} a_{i}^{T} \\
& \mathbf{1}^{T} z=k \\
& 0 \leq z_{i} \leq 1, \quad i=1, \ldots, m
\end{array}
$$

with variables $z \in \mathbf{R}^{m}$ and $X \in \mathbf{S}^{n}$ (set of symmetric $n \times n$ matrices). To form the Lagrangian of the problem (27) we introduced Lagrange multipliers $\lambda_{i}$ for $z_{i} \geq 0, \mu_{i}$ for $z_{i} \leq 1, \nu$ for 
$\mathbf{1}^{T} z=k$, and $\Lambda$ for $X=\sum_{i=1}^{m} z_{i} a_{i} a_{i}^{T}$. The Lagrangian $L$ is

$$
L(z, X, \Lambda, \lambda, \mu, \nu)=\log \operatorname{det} X^{-1}+\operatorname{tr}(X \Lambda)-\sum_{i=1}^{m} z_{i} a_{i}^{T} \Lambda a_{i}+\nu\left(\mathbf{1}^{T} z-k\right)-\lambda^{T} z-\mu^{T}(\mathbf{1}-z),
$$

where $\Lambda \in \mathbf{S}^{n}, \lambda \in \mathbf{R}^{m}, \mu \in \mathbf{R}^{m}$, and $\nu \in \mathbf{R}$. Rearranging the terms we get

$$
L(z, X, \Lambda, \lambda, \mu, \nu)=\log \operatorname{det} X^{-1}+\operatorname{tr}(X \Lambda)+\sum_{i=1}^{m} z_{i}\left(\nu-a_{i}^{T} \Lambda a_{i}-\lambda_{i}+\mu_{i}\right)-k \nu-\mathbf{1}^{T} \mu .
$$

The Lagrange dual function $g$ is given by

$$
g(\Lambda, \lambda, \mu, \nu)=\inf _{z, X} L(z, X, \Lambda, \lambda, \mu, \nu) .
$$

Minimum of $L$ over $z_{i}$ is bounded only if

$$
\nu-a_{i}^{T} \Lambda a_{i}-\lambda_{i}+\mu_{i}=0 .
$$

Minimizing $L$ over $X$ yields $X^{-1}=\Lambda$. The Lagrange dual function $g$ is

$$
g(\Lambda, \lambda, \mu, \nu)= \begin{cases}\log \operatorname{det} \Lambda+n-k \nu-\mathbf{1}^{T} \mu & \nu+\mu_{i}-a_{i}^{T} \Lambda a_{i}=\lambda_{i}, \quad i=1, \ldots, m \\ -\infty & \text { otherwise. }\end{cases}
$$

The dual problem is

$$
\begin{array}{ll}
\operatorname{maximize} & \log \operatorname{det} \Lambda+n-k \nu-\mathbf{1}^{T} \mu \\
\text { subject to } & \nu+\mu_{i}-a_{i}^{T} \Lambda a_{i}=\lambda_{i}, \quad i=1, \ldots, m \\
& \lambda_{i} \geq 0, \quad i=1, \ldots, m \\
& \mu_{i} \geq 0, \quad i=1, \ldots, m
\end{array}
$$

with variables $\Lambda, \lambda, \mu$, and $\nu$. (The constraint $\Lambda \succ 0$, i.e., $\Lambda$ positive definite, is implicit.) The variable $\lambda$ can be eliminated, and we write the dual problem as

$$
\begin{array}{ll}
\operatorname{minimize} & \log \operatorname{det} \Lambda^{-1}-n+k \nu+\mathbf{1}^{T} \mu \\
\text { subject to } & \nu+\mu_{i} \geq a_{i}^{T} \Lambda a_{i}, \quad i=1, \ldots, m \\
& \mu_{i} \geq 0, \quad i=1, \ldots, m
\end{array}
$$

with variables $\Lambda \in \mathbf{S}^{n}, \mu \in \mathbf{R}^{n}$, and $\nu \in \mathbf{R}$.

\section{References}

[BKG06] F. Bian, D. Kempe, and R. Govindan. Utility based sensor selection. In Proceedings of the fifth international conference on Information Processing in Sensor Networks, pages 11-18, Nashville, TN, USA, 2006. 
[BV04] S. Boyd and L. Vandenberghe. Convex Optimization. Cambridge University Press, 2004.

[CHZ02] M. Chu, H. Haussecker, and F. Zhao. Scalable information-driven sensor querying and routing for ad hoc heterogeneous sensor networks. International Journal of High Performance Computing Applications, 16(3):293, 2002.

[CJ95] S. Crary and Y. Jeong. Bayesian optimal design of experiments for sensor calibration. The 8th International Conference on Solid-State Sensors and Actuators, 1995 and Eurosensors IX. Transducers '95, 2, 1995.

[DLT02] R. Debouk, S. Lafortune, and D. Teneketzis. On an optimization problem in sensor selection. Discrete Event Dynamic Systems, 12(4):417-445, 2002.

[Don06] D. Donoho. Compressed sensing. IEEE Transactions on Information Theory, 52(4):1289-1306, 2006.

[DPWH04] A. Dolia, S. Page, N. White, and C. Harris. D-optimality for minimum volume ellipsoid with outliers. Proceedings of the 7th International Conference on Signal/Image Processing and Pattern Recognition, pages 73-76, 2004.

[EFP03] E. Ertin, J. Fisher, and L. Potter. Maximum mutual information principle for dynamic sensor query problems. Proceedings of IPSN, 3, 2003.

[Fed72] V. Fedorov. Theory of optimal experiments. Academic Press New York, 1972.

[FKW03] J. Feldman, D. Karger, and M. Wainwright. LP decoding. Proceedings of the 41st Allerton Conference on Communications, Control, and Computing, pages 1-3, October 2003.

[GBY07] M. Grant, S. Boyd, and Y. Ye. CVX Version 1.1. Matlab Software for Disciplined Convex Programming, 2007. Available at www.stanford.edu/ boyd/cvx/.

[GCHM06] V. Gupta, T. Chung, B. Hassibi, and R. Murray. On a stochastic sensor selection algorithm with applications in sensor scheduling and sensor coverage. Automatica, 42(2):251-260, 2006.

[GJ95] C. Giraud and B. Jouvencel. Sensor selection: A geometrical approach. Proceedings of the IEEE/RSJ International Conference onIntelligent Robots and Systems, 2, 1995.

[GKS05] C. Guestrin, A. Krause, and A. Singh. Near-optimal sensor placements in Gaussian processes. Technical report, School of Computer Science, Carnegie Mellon University, 2005.

[HHB98] A. Hassibi, J. How, and S. Boyd. Low-authority controller design via convex optimization. Proceedings of the 37th IEEE Conference on Decision and Control, $1,1998$. 
[HK93] R. Hettich and K. Kortanek. Semi-infinite programming: Theory, methods, and applications. SIAM Review, 35(5):380-429, 1993.

[HM97] G. Hovland and B. McCarragher. Dynamic sensor selection for robotic systems. Proceedings of the IEEE International Conference on Robotics and Automation, $1,1997$.

[IB05] V. Isler and R. Bajcsy. The sensor selection problem for bounded uncertainty sensing models. In Proceedings of the fourth international symposium on Information Processing in Sensor Networks, Los Angeles, California, 2005.

[JD75] R. John and N. Draper. D-optimality for regression designs: A review. Technometrics, 17(1):15-23, 1975.

[JKG03] S. Jiang, R. Kumar, and H. Garcia. Optimal sensor selection for discrete-event systems with partial observation. IEEE Transactions on Automatic Control, 48(3):369-381, 2003.

[Kam91] D. Kammer. Sensor placement for on-orbit modal identification and correlation of large space structures. Journal of Guidance, Control, and Dynamics, 14:251$259,1991$.

[KP98] M. Kalandros and L. Pao. Controlling target estimate covariance in centralized multi-sensor systems. Proceedings of the American Control Conference, 5, 1998.

[KP99] K. Kookos and J. Perkins. A systematic method for optimum sensor selection in inferential control systems. Industrial Engineering Chemical Research, 38(11):4299-4308, 1999.

[KP02] K. Kincaid and S. Padula. D-optimal designs for sensor and actuator locations. Computers and Operations Research, 29(6):701-713, 2002.

[LFB07] M. Lobo, M. Fazel, and S. Boyd. Portfolio optimization with linear and fixed transaction costs. Annals of Operations Research, 152(1):341-365, 2007.

[LSZ04] Z. Luo, J. Sturm, and S. Zhang. Multivariate nonnegative quadratic mappings. SIAM Journal on Optimization, 14(4):1140-1162, 2004.

[LW66] E. L. Lawler and D. E. Wood. Branch-and-bound methods: A survey. Operations Research, 14:699-719, 1966.

[MB07] A. Mutapcic and S. Boyd. Cutting-set methods for robust convex optimization with worst-case oracles, November 2007. Manuscript, available at www.stanford.edu/ boyd/papers/prac_robust.html.

[MDW95] J. Manyika and H. Durrant-Whyte. Data Fusion and Sensor Management: A Decentralized Information-Theoretic Approach. Prentice Hall PTR Upper Saddle River, NJ, USA, 1995. 
[MN94] A. Miller and J. Nguyen. Algorithm AS 295: A Fedorov exchange algorithm for D-optimal design. Applied Statistics, 43(4):669-677, 1994.

[MR95] R. Motwani and P. Raghavan. Randomized Algorithms. Cambridge University Press, 1995.

[NM92] N. Nguyen and A. Miller. A review of some exchange algorithms for constructing discrete D-optimal designs. Computational Statatistics and Data Analysis, 14:489-498, 1992.

[Osh94] Y. Oshman. Optimal sensor selection strategy for discrete-time state estimators. IEEE Transactions on Aerospace and Electronic Systems, 30(2):307-314, 1994.

[Puk06] F. Pukelsheim. Optimal Design of Experiments. Society for Industrial \& Applied Mathematics, 2006.

$\left[\right.$ REJ $\left.^{+} 07\right]$ H. Rowaihy, S. Eswaran, M. Johnson, D. Verma, A. Bar-Noy, T. Brown, and T. La Porta. A survey of sensor selection schemes in wireless sensor networks. Proceedings of SPIE, 6562, 2007.

[SF04] P. Sun and R. Freund. Computation of minimum-volume covering ellipsoids. Operations Research, 52(5):690-706, 2004.

[Tib96] R. Tibshirani. Regression shrinkage and selection via the lasso. Journal of the Royal Statistical Society. Series B (Methodological), 58(1):267-288, 1996.

[Tro06] J. Tropp. Just relax: convex programming methods for identifying sparse signals in noise. IEEE Transactions on Information Theory, 52(3):1030-1051, 2006.

[VBG97] L. Vandenberghe, S. Boyd, and A. El Gamal. Optimal wire and transistor sizing for circuits with non-tree topology. Proceedings of the 1997 IEEE/ACM International Conference on Computer Aided Design, pages 252-259, 1997.

[Wel82] W. Welch. Branch-and-bound search for experimental designs based on Doptimality and other criteria. Technometrics, 24(1):41-48, 1982.

[Wyn72] H. Wynn. Results in the theory and construction of D-optimum experimental designs. Journal of the Royal Statistical Society. Series B (Methodological), 34(2):133-147, 1972.

[WYPE04] H. Wang, K. Yao, G. Pottie, and D. Estrin. Entropy-based sensor selection heuristic for target localization. In Proceedings of the third international symposium on Information Processing in Sensor Networks, pages 36-45, Berkeley, California, USA, 2004.

[YSK93] L. Yao, W. Sethares, and D. Kammer. Sensor placement for on-orbit modal identification via a genetic algorithm. American Institute of Aeronautics and Astronautics Journal, 31(10):1922-1928, 1993. 
[ZG04] F. Zhao and L. Guibas. Wireless Sensor Networks: An Information Processing Approach. Morgan Kaufmann, 2004.

[ZSR02] F. Zhao, J. Shin, and J. Reich. Information-driven dynamic sensor collaboration for tracking applications. IEEE Signal Processing Magazine, 19(2):61-72, 2002. 